\title{
Networked Microgrids Scoping Study
}

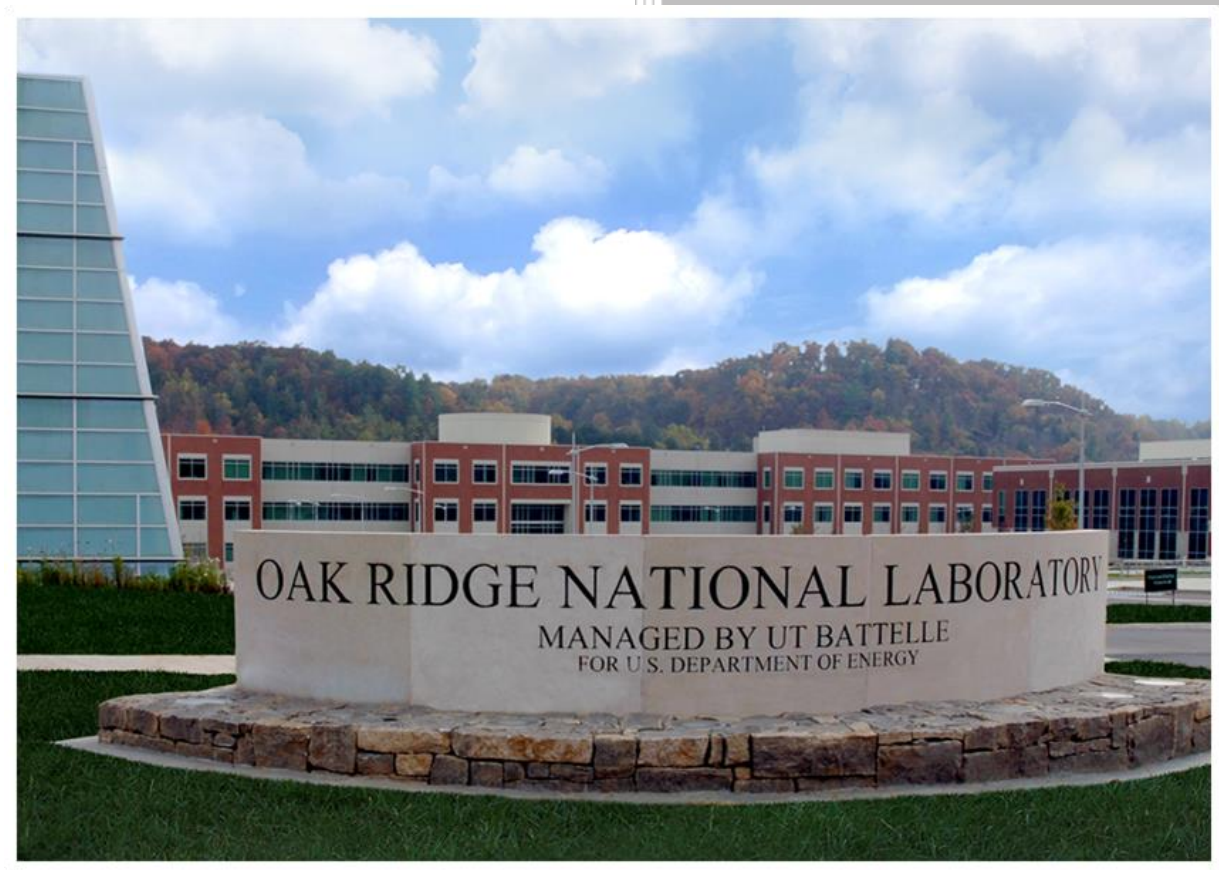

\section{Approved for public release.} Distribution is unlimited.
Guodong Liu Michael R. Starke Ben Ollis Yaosuo Xue October 2016 


\title{
DOCUMENT AVAILABILITY
}

Reports produced after January 1, 1996, are generally available free via US Department of Energy (DOE) SciTech Connect.

Website http://www.osti.gov/scitech/

Reports produced before January 1, 1996, may be purchased by members of the public from the following source:

\author{
National Technical Information Service \\ 5285 Port Royal Road \\ Springfield, VA 22161 \\ Telephone 703-605-6000 (1-800-553-6847) \\ TDD 703-487-4639 \\ Fax 703-605-6900 \\ E-mail info@ntis.gov \\ Website http://www.ntis.gov/help/ordermethods.aspx
}

Reports are available to DOE employees, DOE contractors, Energy Technology Data Exchange representatives, and International Nuclear Information System representatives from the following source:

Office of Scientific and Technical Information

PO Box 62

Oak Ridge, TN 37831

Telephone 865-576-8401

Fax 865-576-5728

E-mail reports@osti.gov

Website http://www.osti.gov/contact.html

This report was prepared as an account of work sponsored by an agency of the United States Government. Neither the United States Government nor any agency thereof, nor any of their employees, makes any warranty, express or implied, or assumes any legal liability or responsibility for the accuracy, completeness, or usefulness of any information, apparatus, product, or process disclosed, or represents that its use would not infringe privately owned rights. Reference herein to any specific commercial product, process, or service by trade name, trademark, manufacturer, or otherwise, does not necessarily constitute or imply its endorsement, recommendation, or favoring by the United States Government or any agency thereof. The views and opinions of authors expressed herein do not necessarily state or reflect those of the United States Government or any agency thereof. 
Electrical and Electronic Systems Research Division

\title{
NETWORKED MICROGRIDS SCOPING STUDY
}

\author{
Guodong Liu \\ Michael R. Starke \\ Ben Ollis \\ Yaosuo Xue
}

Date Published: October 2016

Prepared by

OAK RIDGE NATIONAL LABORATORY

Oak Ridge, TN 37831-6283

managed by

UT-BATTELLE, LLC

for the

US DEPARTMENT OF ENERGY

under contract DE-AC05-00OR22725 



\section{CONTENTS}

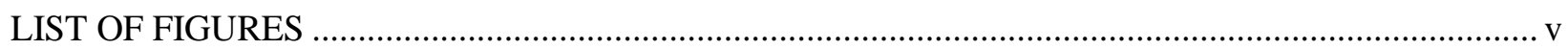

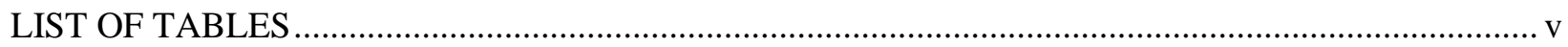

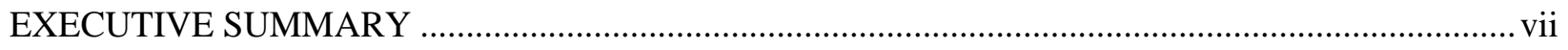

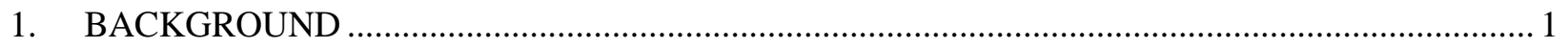

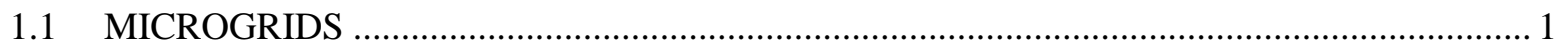

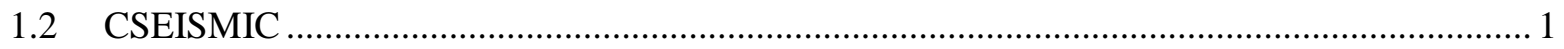

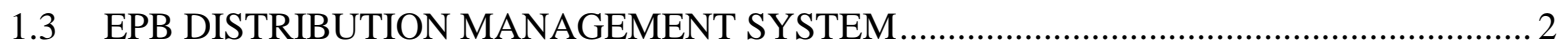

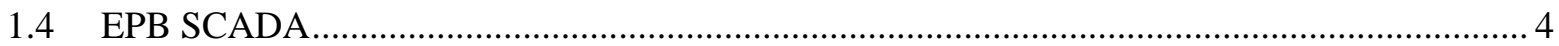

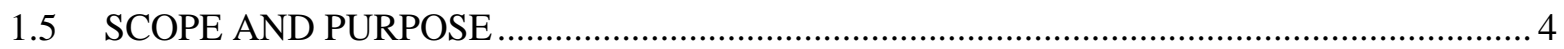

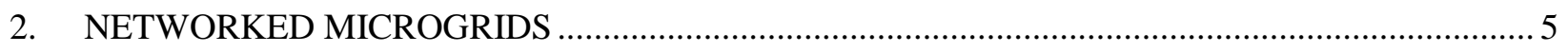

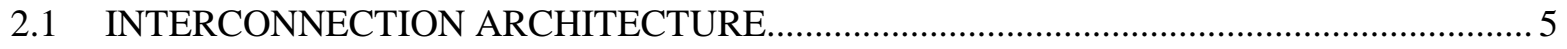

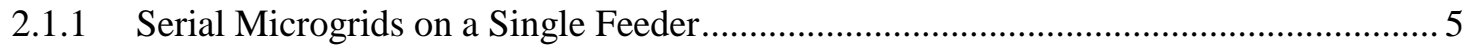

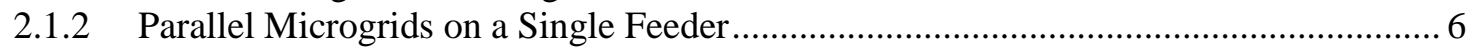

2.1.3 Interconnected Microgrids on Multiple Feeders ..................................................... 7

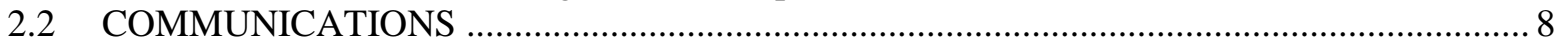

2.2.1 Direct Master-Slave Communications …............................................................ 8

2.2.2 Publish/Subscribe Communications ….............................................................. 9

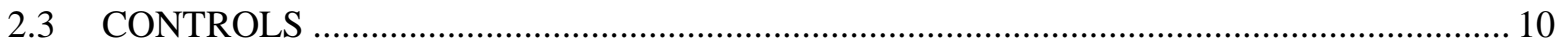

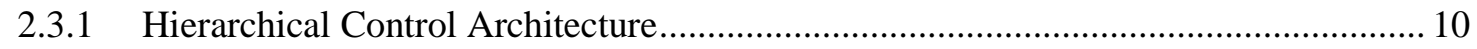

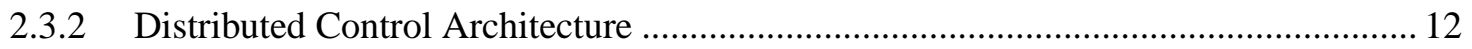

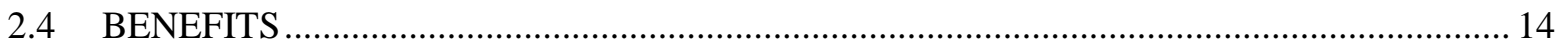

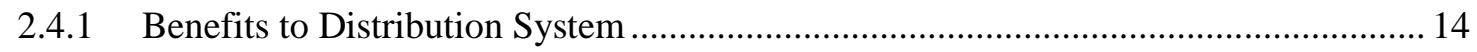

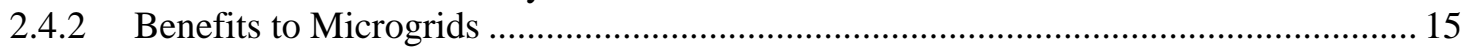

3. FUNCTIONAL REQUIREMENTS OF DMS AND MICROGRID CONTROLLER ....................... 16

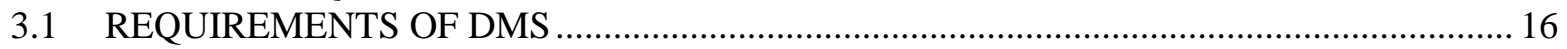

3.2 REQUIREMENTS OF MICROGRID CONTROLLER …............................................ 18

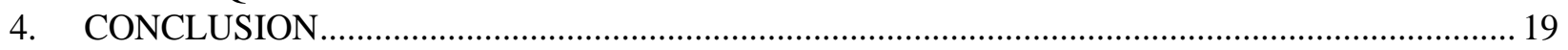

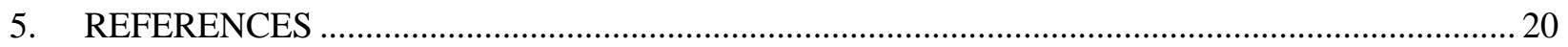





\section{LIST OF FIGURES}

Figure 1. CSEISMIC microgrid control and communication architecture. ......................................... 2

Figure 2. Candidate microgrids integrated with EPB feeders........................................................... 3

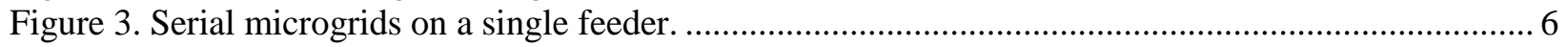

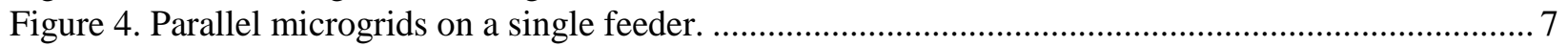

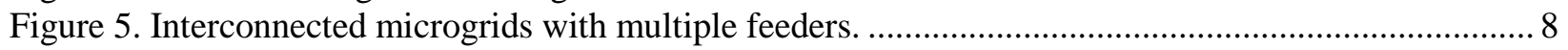

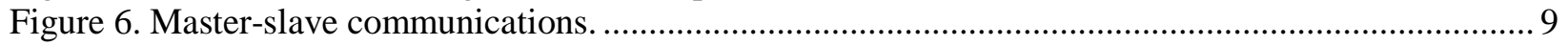

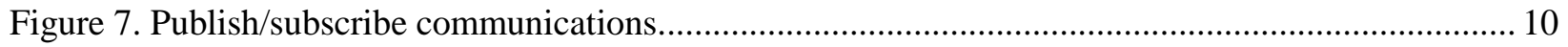

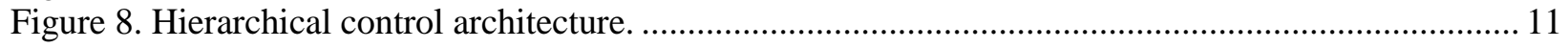

Figure 9. Example of resynchronization under hierarchy control architecture ........................................ 12

Figure 10. Distributed control with publish/subscribe communications................................................. 13

Figure 11. Example of resynchronization under distributed control architecture.................................... 14

\section{LIST OF TABLES}

Table 1. Functional requirements for DMS integration of microgrids and DERs ................................ 17

Table 2. Functional requirements for microgrid controller..... 



\section{EXECUTIVE SUMMARY}

While the utilization of a microgrid for local power reliability during grid outage and emergencies is a well-known benefit, the integration of microgrids with the broader electrical distribution system will allow for seamless interaction with distribution system operations, contributing to resource and economic optimization, enhanced reliability and resiliency, and improved power quality. By virtue of integration with the distribution system, multiple microgrids should be networked and collectively known as networked microgrids.

As a follow-up to the work conducted by Oak Ridge National Laboratory on a microgrid controller [the Complete System-level Efficient and Interoperable Solution for Microgrid Integrated Controls (CSEISMIC)], the main goal of this work is to identify the next steps for bringing microgrid research to the utility industry, particularly as a resource for enhancing efficiency, reliability, and resilience. Various $R \& D$ needs for the integration of microgrids into the distribution system have been proposed, including interconnection types, communications, control architectures, quantification of benefits, functional requirements, and various operational issues.

The main contributions of this report are as follows:

1) Three basic interconnection layouts of networked microgrid, including serial microgrids on a single feeder, parallel microgrids on a single feeder, and interconnected microgrids on multiple feeders, are proposed. The unique requirements for operation of each layout are presented.

2) The communication and control architectures for networked microgrids are described. The direct master-slave communication and publish and subscribe communication are presented separately. Two basic control architectures, hierarchical and distributed control, are described and compared.

3) The potential benefits from networked microgrids to both the distribution system and the microgrids are discussed. To realize these benefits, the functional requirements of distribution management systems (DMSs) and microgrid controllers are presented.

Several microgrid research needs were determined in this scoping:

- Coordination of

- energy trading and ancillary service management

- microgrids/distributed energy resources with feeder reconfiguration/restoration

- protection scheme (from islanded individually to various subnetwork connections)

- Higher order power balancing of local imbalances to compensate for a variable supply of renewable energy

- Multiobjective operational optimization (peak shaving, loss minimization, voltage regulation) across several microgrids

- Aggregated uncertainty management

- Jurisdiction and dynamic boundaries among microgrid energy management systems (EMSs), networked microgrid EMSs, and DMSs

- Strong interaction between active power and reactive power schedule and control 
- Quantification and allocation of the benefits of networked microgrids

- Distribution energy market managed by distribution system operator (DSO)

- Distribution ancillary service market managed by DSO

- Pricing mechanism in distribution network and impact on microgrid economics and optimization

- Demand response incentive signal design

- Defining communication topics for microgrid-to-microgrid communications

- Demonstrable networked microgrid communications 


\section{BACKGROUND}

\subsection{MICROGRIDS}

The growth of distributed renewable and/or nonrenewable energy resource installations, emerging utilityscale energy storage, plug-in hybrid electric vehicle (PHEV) utilization, and demand response is bringing unprecedented opportunities and challenges to the electric distribution system. As these technologies evolve, utilities, end users, manufacturers, and other participants in distribution system operations are actively transforming the utility operational model. In the past, utility systems were able to mostly operate in isolation, but they are now facing a systems integration challenge that requires complex coordination and integration of these distributed resources.

One available approach to integrate these technologies is through a microgrid. As defined by the Microgrid Exchange Group, "A microgrid is a group of interconnected loads and distributed energy resources within clearly defined electrical boundaries that acts as a single controllable entity with respect to the grid. A microgrid can connect and disconnect from the grid to enable it to operate in both gridconnected or island-mode [1]." This definition of microgrid has been adopted by the U.S. Department of Energy as well as by the Electric Power Research Institute. By this definition, a microgrid can be regarded as a single controllable resource connected to a distribution network.

With a microgrid, power can be imported or exported to the main distribution network, depending on the system constraints and economic incentives (market tariffs). In addition, a microgrid can provide ancillary services such as voltage support and regulation services in support of the main distribution grid or utility. This is a feature that a conventional end-user system cannot deliver [2], [3]. Furthermore, a microgrid not only provides energy; it also improves local reliability, reduces emissions, and contributes to a lower cost of energy supply by taking advantage of distributed energy resources (DERs), storage devices, and responsive loads [4]. A microgrid can also improve power quality by supporting voltage and reducing voltage dips within the microgrid [5]. These benefits also support the end-use customers and are attracting increasing attention from both academia and industry [6].

\subsection{CSEISMIC}

Complete System-level Efficient and Interoperable Solution for Microgrid Integrated Controls (CSEISMIC) is a microgrid control solution with both real-time operation and control functions and capabilities of participating in the energy and ancillary markets. The system diagram and communication

links are illustrated in Figure 1. The first release of CSEISMIC is as a central controller which consists of two parts, an energy management system (EMS) and a supervisory control and data acquisition system (SCADA). The SCADA system communicates with all the devices in a microgrid (including the microgrid switch, energy resources, loads, and protection relays); collects the real-time microgrid data for visualization and historical tracking; and sets the operation modes for the generation resources and loads. SCADA also communicates with the EMS to send microgrid measurement data and receive optimization dispatch commands. The EMS optimizes power dispatches to meet operational objectives and balances these objectives with commands received from the distribution system operator to provide requested energy and/or ancillary services. Therefore, CSEISMIC is not only the control and management center of the microgrid; it is also the interface between the distribution management system (DMS) and the microgrid. 


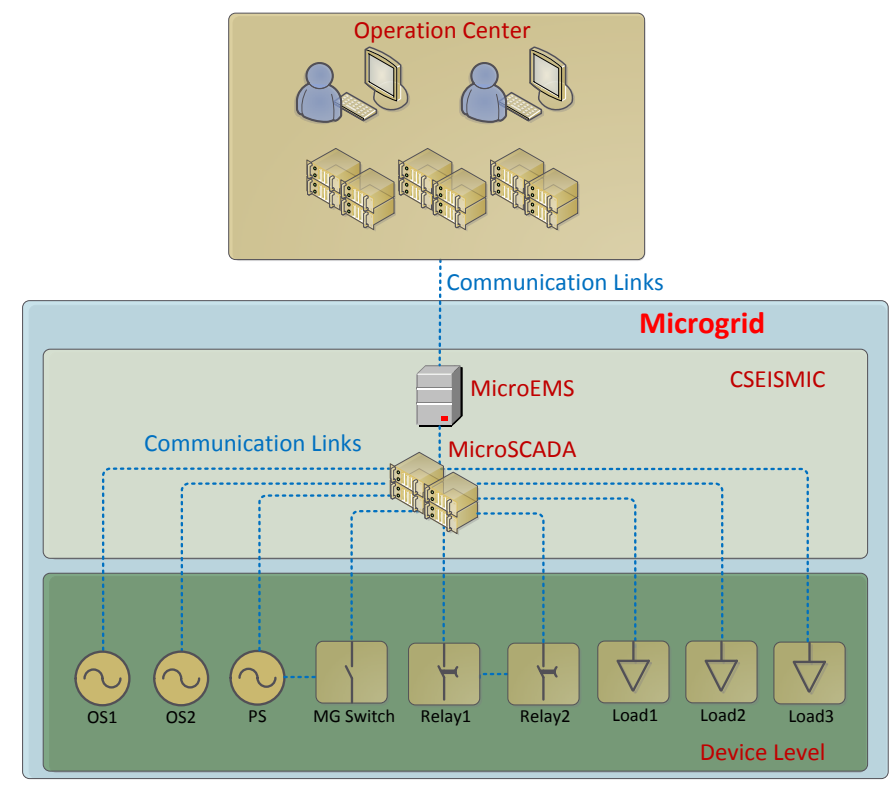

Figure 1. CSEISMIC microgrid control and communication architecture.

The operational states of generation assets are governed by the control philosophy determined in the design framework of the microgrid controller. For the initial development of CSEISMIC, a master-slave type architecture has been implemented [7]. In this architecture, the generation assets are controlled in one of three states: voltage and frequency (V/f), real and reactive power $(\mathrm{P} / \mathrm{Q})$, or real power and voltage $(\mathrm{P} / \mathrm{V})$. While connected to a larger grid, all of the sources are controlled in either $\mathrm{P} / \mathrm{Q}$ or $\mathrm{P} / \mathrm{V}$. The grid provides the frequency reference. In islanded operation mode, one of the sources must take the responsibility for providing the frequency reference as a V/f source while other generation assets behave as either $\mathrm{P} / \mathrm{Q}$ or $\mathrm{P} / \mathrm{V}$ sources. The transient control and state changes to maintain stability during islanding and resynchronization are some of the main challenges in microgrid operation.

The first generation of CSEISMIC uses the Modbus communication protocol. This protocol is widely accepted by industry and is often used in various systems such as energy storage and diesel generators (where a communications interface is available). The CSEISMIC implementation has been programmed in the LabVIEW real-time layer using a set of Modbus addresses defined by the Oak Ridge National Laboratory team for demonstration purposes. The communication speed is on the order of cycles, and round trip delays of 5-6 cycles $(60 \mathrm{~Hz})$ have been recorded for islanding and resynchronization. (The islanding switch changes the state; the master controller notifies the V/f source of a needed state change; and the time will vary based on distance). At this time, the communication system between the SCADA system of the Electrical Power Board (EPB) of Chattanooga and the field devices is primarily based on DNP3 (Distributed Network Protocol 3). For integration of CSEISMIC into EPB SCADA, an adapter will be created to communicate between EPB and CSEISMIC. This report defines the communication data and requests that will be needed.

\subsection{EPB DISTRIBUTION MANAGEMENT SYSTEM}

The DMS implemented at EPB was developed by Open Systems International, Inc. (OSI). The software suite being used is Spectra Suite, OSI's DMS. This software is a powerful set of tools that allow utility operators to view and manage their distribution network, from topology and power flow to automation and switch order management. The OSI Spectra Suite consists of several useful products. Spectra Distribution Power Flow ${ }^{\mathrm{TM}}$ is a three-phase distribution network power flow calculation tool. Spectra 
Voltage/VAR Control ${ }^{\mathrm{TM}}$ is a voltage and VAR control tool for loss reduction, conservation voltage reduction (CVR), and power factor improvement. Spectra Fault Location, Isolation, and Service Restoration $^{\mathrm{TM}}$ is a fault location, isolation, and service restoration tool. Finally, Spectra Feeder Reconfiguration $^{\mathrm{TM}}$ is a feeder reconfiguration tool for loss reduction and voltage profile improvement.

Currently, seven functions have been fully implemented at EPB:

- unbalanced three-phase power flow (basic model and tool),

- Volt/VAR control (voltage reduction as a special case),

- fault location,

- fault analysis,

- service restoration,

- feeder reconfiguration, and

- planned switching.

These functions work together to automatically manage the distribution network operation, improve reliability, and reduce operational costs for EPB and EPB electricity customers.

At this time, EPB has not had any DERs to include in the DMS. However, a large (greater than $1 \mathrm{MW}$ ) photovoltaic (PV) project is under development, as is an energy storage system $(100 \mathrm{~kW})$. These assets will require adaptation to the EPB DMS or the use of a microgrid controller such as CSEISMIC. Figure 2 shows a preliminary sketch of the installation of the PV and nearby loads. An energy storage system on the same circuit as the PV is expected.

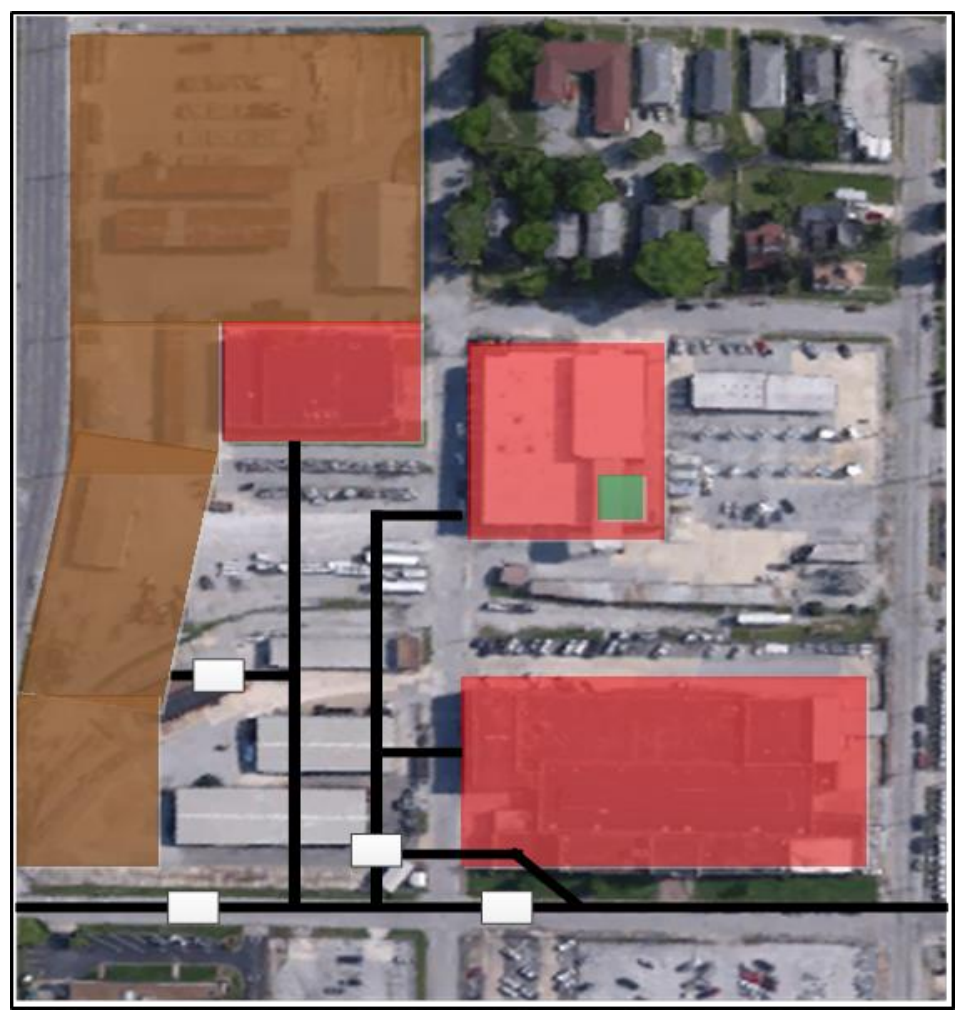

Figure 2. Candidate microgrids integrated with EPB feeders. 


\subsection{EPB SCADA}

EPB's SCADA system has a primary master software system consisting of the OSI Monarch ${ }^{\mathrm{TM}}$ - a collection of applications and servers that acts as a centralized system for controlling and monitoring the EPB distribution system. The suite of applications for SCADA is known as OpenSCADA.

Currently, the EPB SCADA system performs some core capabilities required for electric grid operation, including:

- communicating to and gathering data from field devices;

- reporting the most important grid and device data back to operators;

- real-time visualization of the power system;

- $\quad$ informing operators when abnormal conditions are present; and

- allowing operators to remotely control devices such as breakers, voltage regulators, and capacitor banks.

Communication between SCADA and field devices [such as circuit breakers, transformers, pulse check closing reclosers (PCRs), regulators, load tap changers (LTCs), motor operated switches (MOSs), capacitor banks, and automated switch gear] is primarily performed through DNP3 via the copper (inside the substation) and fiber communication pathways. SCADA polls devices every 2 seconds, but only reports devices with changes in either basic status information or analog data since the last data acquisition. All system data is polled every $5 \mathrm{~min}$ to confirm system integrity regardless of any change observed.

Along with requests for data, the SCADA system can send requests for control actions. SCADA sends the requests to the central processing node (CPN), which then pass the request to the distributed processing nodes (DCNs). The requests could be for the opening of a closed breaker, an increase in the voltage on the LTCs, or change in the PCR operational mode from normal to storm latch. Devices that could be controlled include circuit breakers, PCRs, MOSs, capacitor banks, regulators, LTCs, and ground and reclosing relays. The CPN will also confirm whether the control command was successfully completed by checking the status of the device. The CPN is also the timekeeper and passes time from the GPS to the DCN.

\subsection{SCOPE AND PURPOSE}

With the increasing incorporation of DERs, energy storage, PHEVs, and demand response into distribution systems, the traditional passive distribution networks are gradually being transformed into active systems composed of multiple microgrids. However, to best integrate multiple microgrids into an electrical distribution system, understanding the benefits, needs, and challenges of networking the microgrids and interconnecting these to the utility DMS is a vital topic of research and development.

Intuitively, each microgrid has a microgrid controller to coordinate the energy consumption or production of the generation assets. However, participation in electricity/ancillary service markets to maximize benefits requires coordination of the microgrid controllers and utility DMS to make this a functional benefit. Furthermore, coordination of the interconnection in terms of islanding, resynchronization, optimization, and other functions among the microgrids and DMS will play a crucial role in assuring that microgrid stability and economic objectives are reached. 
This report discusses the feasibility of integrating networked microgrids into a utility distribution system. Several architectures are proposed and used to drive key questions of the functional requirements of both microgrid controllers and DMS use. In addition, the possible benefits as well as operational issues of interconnecting multiple microgrids within a utility are discussed.

\section{NETWORKED MICROGRIDS}

\subsection{INTERCONNECTION ARCHITECTURE}

Recently, interconnected and networked microgrids have become an emerging grid design feature that can provide a number of benefits including economic optimization, reliability, resiliency, and recovery to local distribution networks and customers. The types and methods of interconnections will be numerous, and understanding the challenges created by these interconnected microgrids is of fundamental importance. However, analyzing all the possible variations of multiple microgrids is an impossible undertaking. Hence, the approach used for this report is to break the design for potential microgrids into basic building blocks of two networked microgrids as fundamental components to the network microgrid architecture. As a result, three basic networked microgrid architectures are considered here: serial microgrids on a single feeder, parallel microgrids on a single feeder, and interconnected microgrids with multiple feeders. Each of these configurations poses unique sets of requirements for communications, controls, and needs.

\subsubsection{Serial Microgrids on a Single Feeder}

The serial microgrids on a single feeder configuration consists of two or more microgrids that are interconnected, but only have one direct interface to the utility (Figure 3). This configuration provides only a single direct connection to the utility through Microgrid A (MCA represents the microgrid controller A for Microgrid A). For this example, the communication protocol and controls are assumed to be of the master-slave architecture or hierarchical communications and controls, where the DMS provides communication requests to the master controller of microgrid A, and MCA provides communication requests to MCB (similar to CSEISMIC) for action. Since only one interconnection to the utility is available, any utility DMS does require a communication exchange to the MCB.

In this case, the microgrid interconnections to the distribution system have the following options:

1. Both microgrid A and B are grid-connected with the distribution feeder. Each microgrid controller controls and optimizes the respective microgrid assets based on the information/orders from DMS or request from neighboring microgrids.

2. Both microgrid A and B are islanded. Each microgrid controller controls and optimizes its respective microgrid assets based on internal information and resources.

3. Microgrid A is grid-connected, but microgrid B is islanded. MCA controls microgrid A based on the information/orders from DMS, while MCB controls microgrid B based on internal information and resources.

4. Microgrid A is islanded and microgrid B is interconnected to Microgrid A. Under hierarchical control architecture, microgrid A will either be able to include microgrid B as part of their assets in terms of optimization individually or provide microgrid $\mathrm{B}$ with an overall cost objective and restriction on point of common coupling (PCC) AB voltage and power, etc. Under this situation, MCA should have plug-and-play capability. While under distributed control architecture, MCA and MCB negotiate the restrictions on $\mathrm{PCC} \mathrm{AB}$ voltage and power until reaching an agreement. 


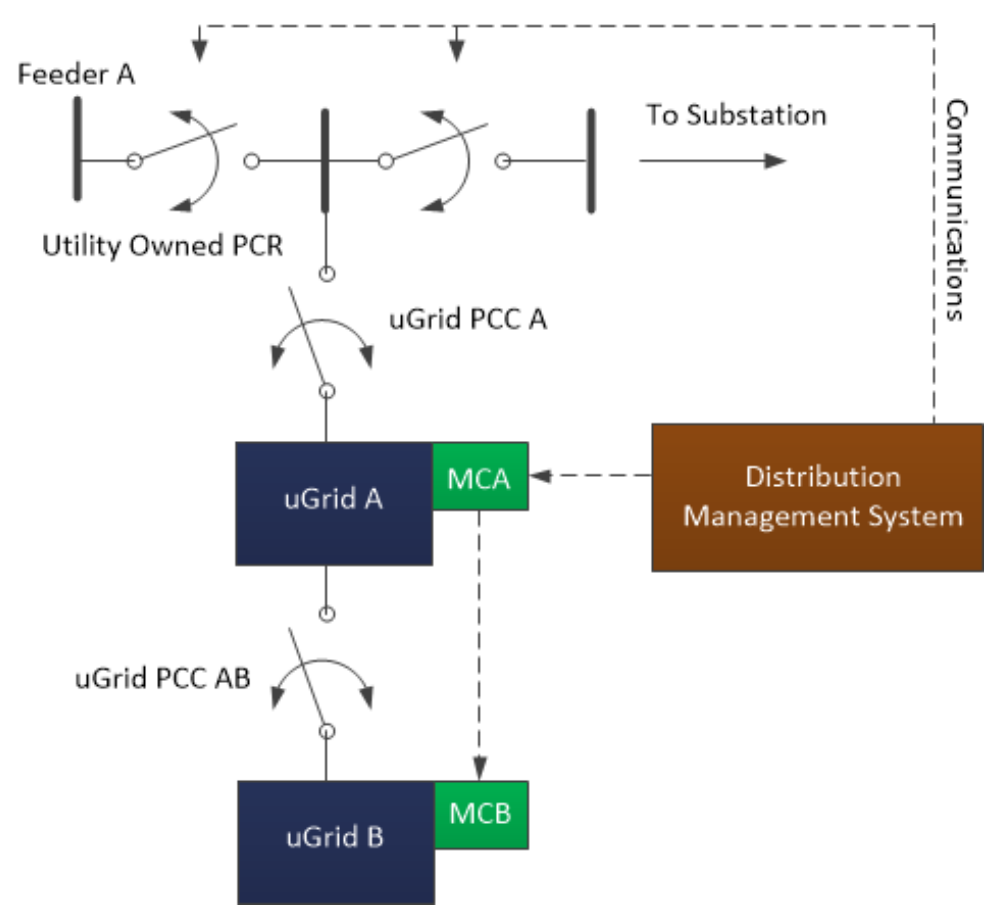

Figure 3. Serial microgrids on a single feeder.

\subsubsection{Parallel Microgrids on a Single Feeder}

The parallel microgrids on a single feeder configuration is two or more microgrids connected separately to a single feeder with a possible interconnection tie (Figure 4). A typical communication linkage of master-slave architecture is shown. In this case, the microgrid interconnections to the distribution system have the following options:

1. Both microgrid A and B are separately grid-connected with the distribution feeder. Each microgrid controller controls and optimizes the respective microgrid assets based on the information/orders from DMS.

2. Both microgrid A and B are islanded. Each microgrid controller controls and optimizes the respective microgrid assets based on internal information and resources.

3. Microgrid A is grid-connected, but microgrid B is islanded, or vice versa. MCA controls microgrid A based on the information/orders from DMS, while MCB controls microgrid B based on internal information and resources.

4. Microgrid A or microgrid B is grid-connected; the other microgrid is interconnected through PCC $\mathrm{AB}$. This option turns to the serial microgrids on a single feeder configuration. 


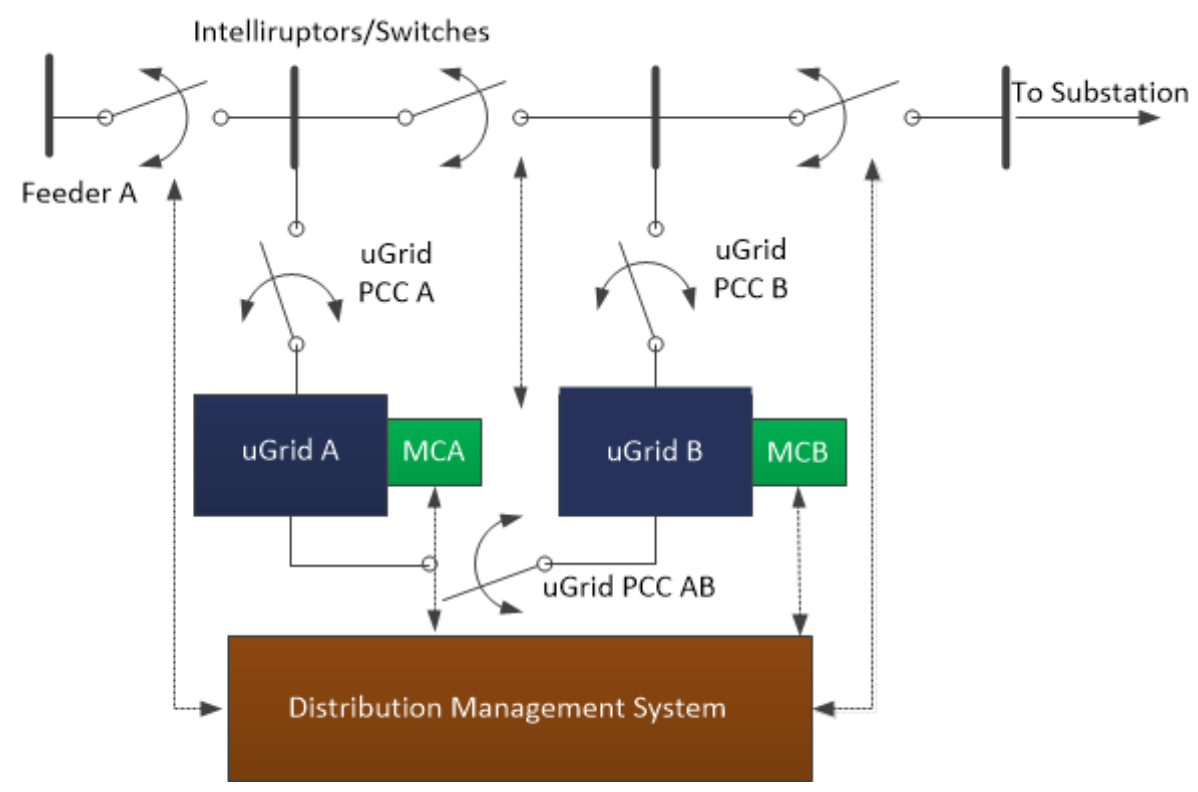

Figure 4. Parallel microgrids on a single feeder.

\subsubsection{Interconnected Microgrids on Multiple Feeders}

The interconnected microgrids on multiple feeders configuration is two or more microgrids connected separately to different feeders with a possible interconnection tie as Figure 5. A typical communication linkage of master-slave architecture is shown. In this case, the microgrid interconnections to the distribution system have the following options:

1. Both microgrid A and B are separately grid-connected with different feeders. Each microgrid controller controls and optimizes the respective microgrid assets based on the information/orders from DMS.

2. Both microgrid A and B are islanded. Each microgrid controller controls and optimizes the respective microgrid assets based on internal information and resources.

3. Microgrid A is grid-connected, but microgrid B is islanded, or vice versa. MCA controls microgrid A based on the information/orders from DMS, while MCB controls microgrid B based on internal information and resources.

4. Microgrid A or microgrid B is grid-connected; the other microgrid is interconnected through PCC $\mathrm{AB}$. The interconnection PCC AB can be used as an interconnection switch. Coordinated with the sectionalizing switches in feeders, additional routes are provided for the restoration of feeders. This is a unique characteristic. 


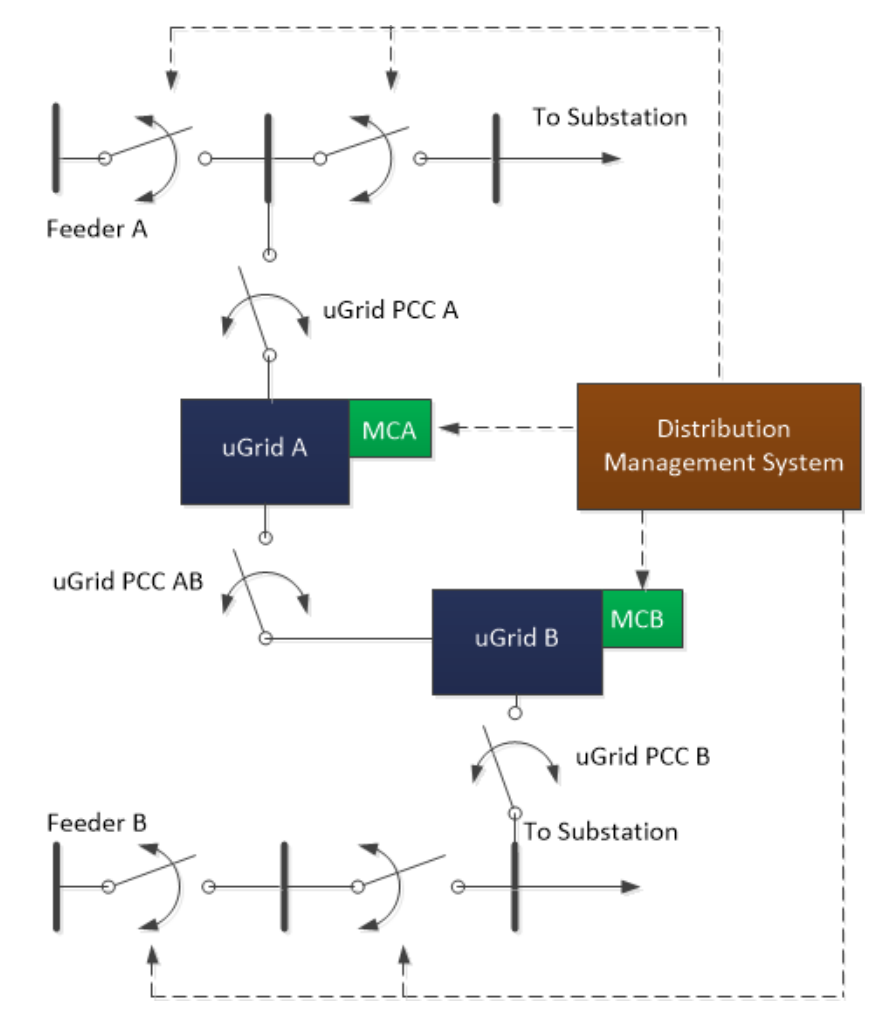

Figure 5. Interconnected microgrids with multiple feeders.

\subsection{COMMUNICATIONS}

While there are numerous communication methods and protocols in the current implementations of the smart grid, this discussion will focus on direct master-slave communications and publish/subscribe communications. For networked microgrids, the communication network enables the DMS, microgrid master controllers, and microgrid devices to intercommunicate. Communications provide a number of important features that otherwise might not be available, including many of the needed functions within a microgrid, coordination of the DMS and microgrids, and microgrid-to-microgrid coordination.

\subsubsection{Direct Master-Slave Communications}

With the direct master-slave type communications, device communication is typically orchestrated by the master initiating a request and a slave providing the requested information or adjustment. Depending on the slave configuration, the master can poll the slave (read the registers) to obtain measurement values or device parameters. The master can also modify the registers of the slave (write the registers) for adjusting parameters or settings or modify control targets. For general microgrid operations, the reading and writing of data from registers occurs on the time interval of seconds. The microgrid controller is the communications director and behaves as the master for all communications.

For the process of islanding and resynchronization, fast communications between the islanding switch, the microgrid controller, and the generation source controller are needed. The microgrid controller is the general coordinator of information, notifying the generation source controllers of any needed state changes due to a state change at the PCC. Other options do exist such that the microgrid controller is purely an initiator of the islanding and resynchronization. However, this is highly complex such that device-to-device communications become necessary, requiring unique configuring of master/slave configurations for communications. For a master controller coordinating the islanding, direct polling of 
the microgrid PCC must occur at a fast rate (ideally less than a cycle). This is needed to ensure that the master controller can deliver the information to the generation source controller as quickly as possible. The speed of the transition governs whether a frequency or voltage excursion on the microgrid occurs, potentially causing devices within the microgrid to trip.

For this type of communication, information is necessary to create the communication gateways. Some examples include IP address for TCP/IP connections and communication ports for serial and TCP/IP connections. As a result, predefined information regarding the connections for the device messaging is required; this is not a "plug-and-play" communications implementation. Some examples of master-slave communications include Modbus, IEC61850 Manufacturing Message Specification, and DNP3. In the first release of CSEISMIC, communications to the devices was through Modbus, and configuration files were used to define these communication interconnections.

For communications between DMS and microgrid controllers, the DMS is the master coordinator, and it provides the instructions down to the Microgrid controllers as slaves (Figure 6). This is the most straightforward approach, but it requires that any needed information between two adjacent microgrids be consistently polled in case information sharing between microgrids is needed. Furthermore, in the case of sharing microgrid assets between one microgrid and another (serial microgrids on a single feeder), this shared information must already pre-exist on the microgrid controller. This creates a complicated set of interlinkages between microgrid controllers that will likely rule out this type of design.

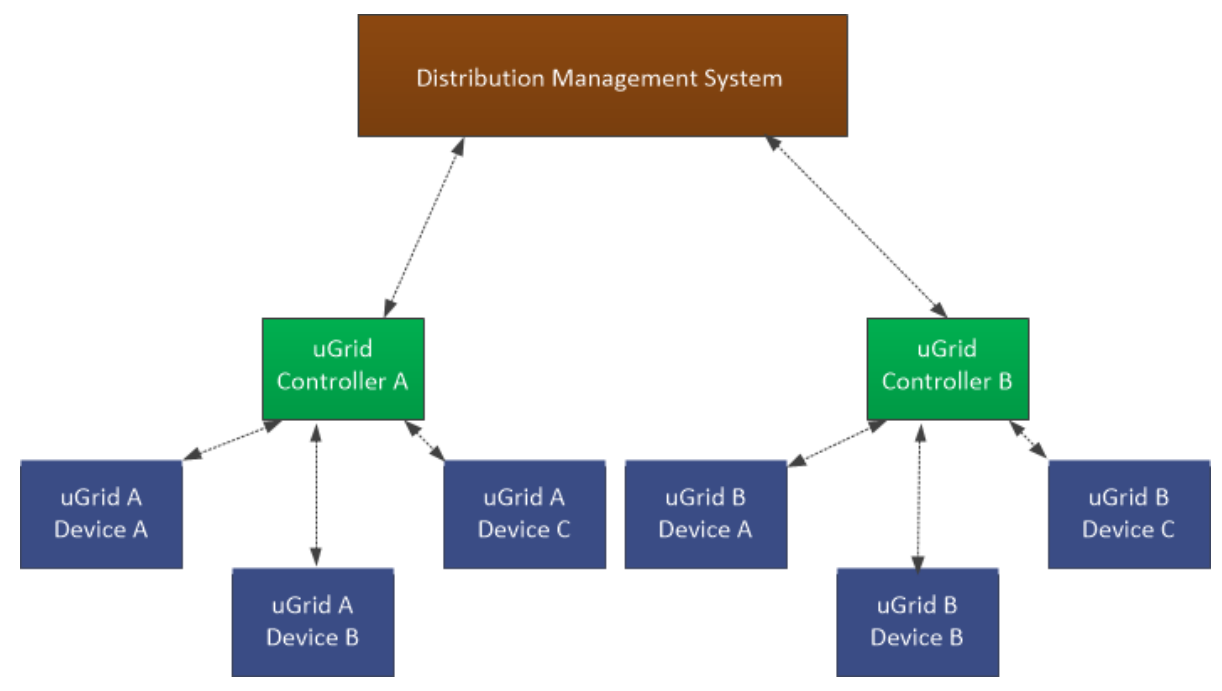

Figure 6. Master-slave communications.

\subsubsection{Publish/Subscribe Communications}

In the publish/subscribe convention, devices broadcast information (publisher) and read broadcast data (subscribers). This requires that devices subscribe to specified topic names as identified per a standard or convention for the communications protocol. Any device can subscribe to any set of information. Hence, islanding and resynchronization can occur independently of any master controller interface (i.e., PCC communicates directly with source controller to state transition). For this communication to be more effective, prioritization of specific communication requests is needed and should still ensure that the communications between the PCC and source controller is fast.

Unlike with master-slave communications architecture, knowledge of the devices that are subscribers to the published information is unnecessary. As a result, no identification regarding the devices is hosted at the primary controller or on any other device. This type of communication provides an opportunity for 
plug-and-play architectures. Some examples of publish/subscribe communications include Data Distribution Service (DDS), Generic Object Oriented Substation Events, and Advanced Messaging Queuing Protocol. In the second release of CSEISMIC, the objective is to use a DDS communication system and devise a plug-and-play approach.

For communications between DMS and microgrid controllers, the DMS could still be the master coordinator and provide the instructions down to the Microgrid controllers as slaves (Figure 7). However, microgrid-to-microgrid communications could be through the publish/subscribe architecture which would significantly reduce significantly reduce the complexity in microgrid-to-microgrid communications. The challenge of performing serial microgrids on a single feeder is reduced as new devices integrated into a higher order microgrid could subscribe to the needed information.

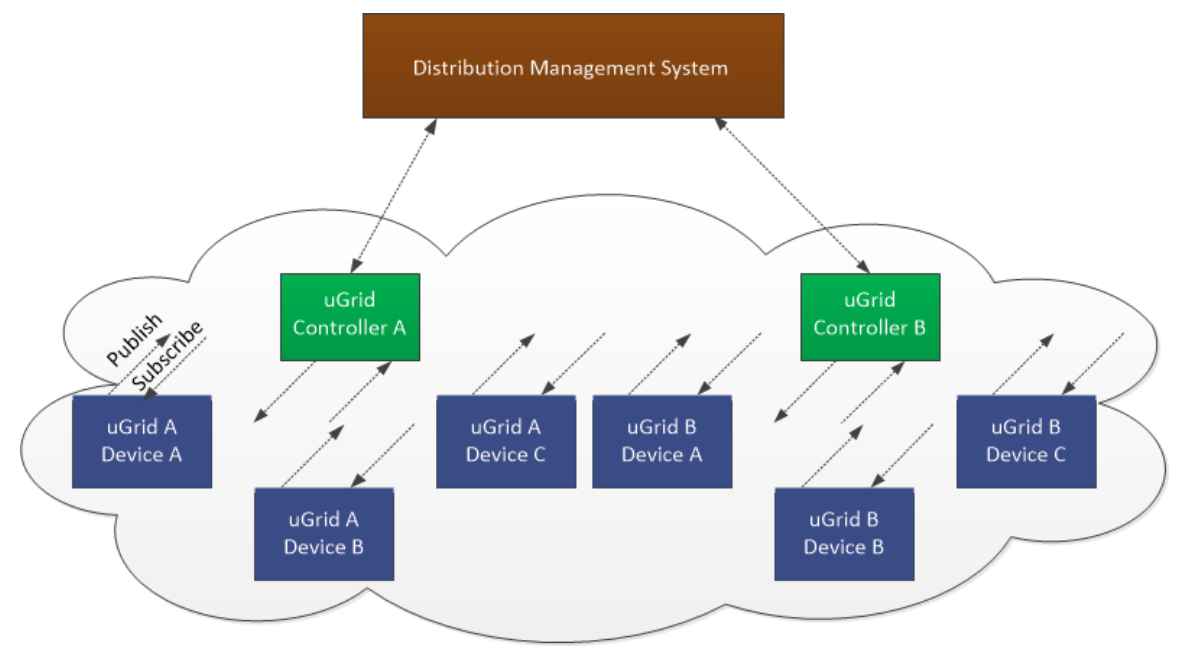

Figure 7. Publish/subscribe communications.

\subsection{CONTROLS}

In this report, the control architecture of networked microgrids is divided into hierarchical control and distributed control. Hybrid control architecture may also be available considering the various ownerships of microgrids, but hybrid control is not discussed here.

\subsubsection{Hierarchical Control Architecture}

Normally, DMS/Advanced DMS (ADMS) or even a third party should support coordination of integrated microgrids under hierarchical control architecture. In this architecture, the power electronics systems provide direct device control with V/f, $\mathrm{P} / \mathrm{Q}$, or $\mathrm{P} / \mathrm{V}$ as the primary control. The microgrid controller provides coordination of these device controls as the secondary control. The DMS provides the final level of coordination as the tertiary control. DMS directly controls all sectionalizing switches as well as microgrid PCCs through microgrid controllers (Figure 8).

- In grid-connected mode, all microgrids are integrated directly with the distribution feeders or other grid-connected microgrids. DMS/ADMS communicates with all microgrids, collects real-time measurements, and send guidelines or orders for each microgrid controller. Then microgrid controllers perform internal optimization of resources to meet needs and/or orders. DMS/ADMS guides the operation of each microgrid using the following options:

- sending individual microgrid price or incentive signals, 
- $\quad$ sending overall microgrid targets/requirements for optimization, or

- with permission, working as a surrogate of the microgrid controller and sending dispatch orders for each resource in the microgrid.

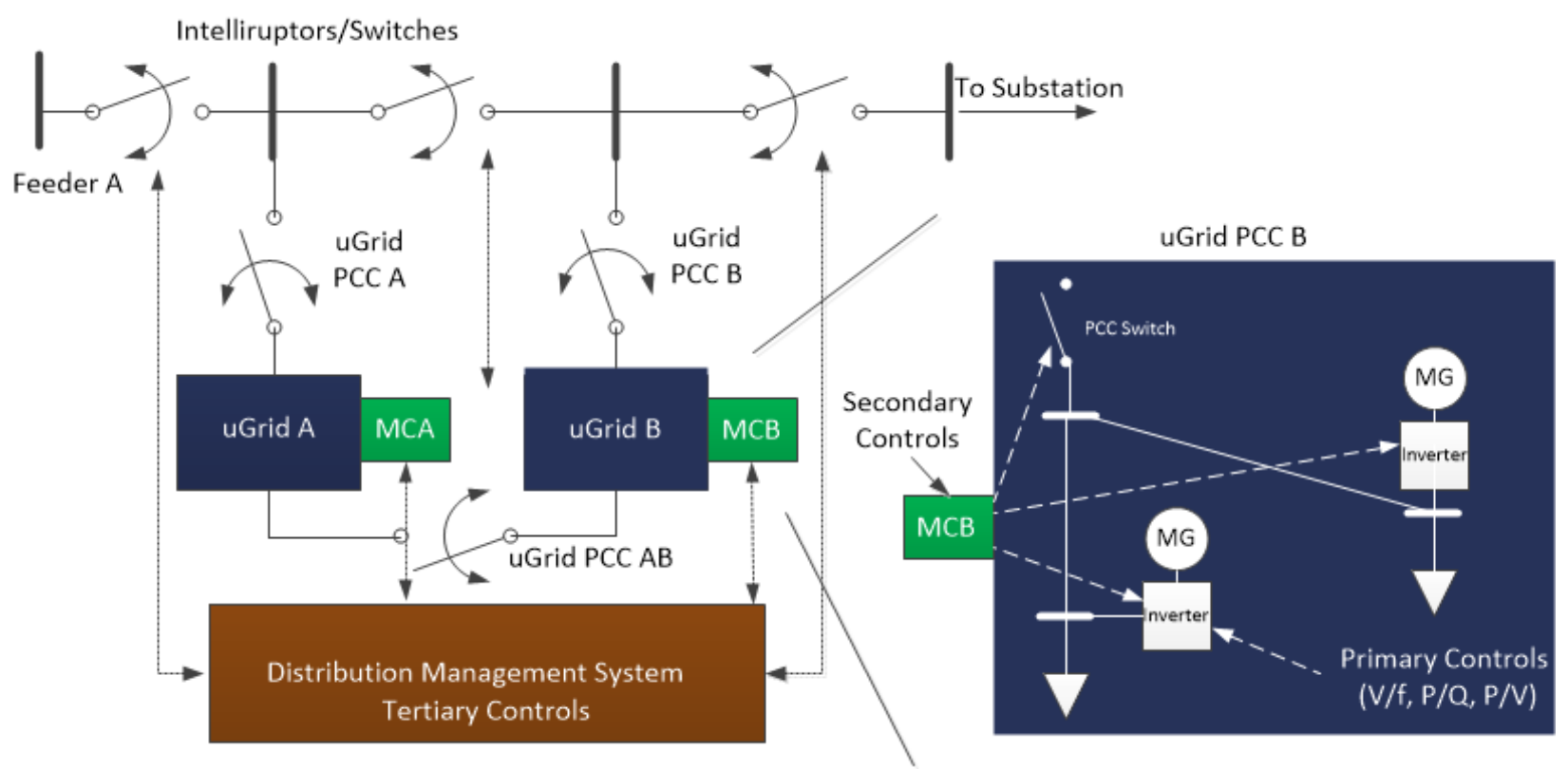

Figure 8. Hierarchical control architecture.

- In islanding mode, each microgrid is required to have a capable V/f source for basic off-grid functionality with a separate microgrid controller. For intentional islanding, DMS/ADMS sends the microgrid controller an island command, and the microgrid controller automatically performs typical microgrid islanding functions with microgrid PCC. Microgrid controllers may also send intentional islanding requests to DMS/ADMS before islanding. For unintentional islanding, a microgrid automatically islands upon disturbance on feeders, and then notifies DMS/ADMS of an unintentional islanding. In this case, DMS/ADMS may automatically request other neighboring microgrids to island as well.

- For the resynchronization process, microgrids should resynchronize to a grid-connected feeder as the first option for economic and reliability benefits. However, if a grid-connected feeder is not available at that moment, multiple neighboring microgrids should merge for better performance. The resynchronization processes for these two options are the following:

- Resynchronization to grid-connected feeder as normal.

- Resynchronization to another islanded microgrid is actually more complicated and challenging. First, the DMS/ADMS must coordinate with the microgrid ensure enough reserve and inertia is available to accommodate the interconnection. If there is no direct PCC switch connection between these microgrids, DMS/ADMS must identify possible routes through available feeders and ensure that the switch between microgrids is closed and that the other switches connected to the distribution feeder are open (Figure 9). This case would most likely occur during the restoration of a distribution grid. This requires strict coordination between microgrid controllers and DMS/ADMS. After both microgrids and DMS are ready for the interconnection, the resynchronization switch is closed, and two islanded microgrids are merged. In the example, 
microgrid B performs a transition to grid connection mode, and the generation resource performing V/f control changes state to P/Q control. For control and optimization of networked microgrids in islanding mode, DMS/ADMS must communicate with these microgrids, collect real-time measurements, and send guidelines or orders for each microgrid controller. Then microgrid controllers perform internal optimization of resources to meet needs and/or orders. The individual microgrid controller could be hierarchical or distributed. The challenges of this kind of resynchronization include:

- The microgrids that will be merged should have enough reserve and inertia to accommodate the new interconnected microgrid. The resynchronization onto a very weak grid (another microgrid) may impact the local voltage at the PCC enough to reinitiate an unintentional island based on settings. CSEISMIC reduces this impact by holding the devices to the current state after interconnection to reduce transient impact by switching into a new system.

- The microgrid controller should dynamically inherit resources (loads, distributed energy resources, switches, etc.) from feeders on the interconnection route or other microgrids. Because the interconnection of islanded microgrids may involve sections of feeders, the boundaries of microgrids are subject to change. This requires that the microgrid controller have plug-and-play capability.

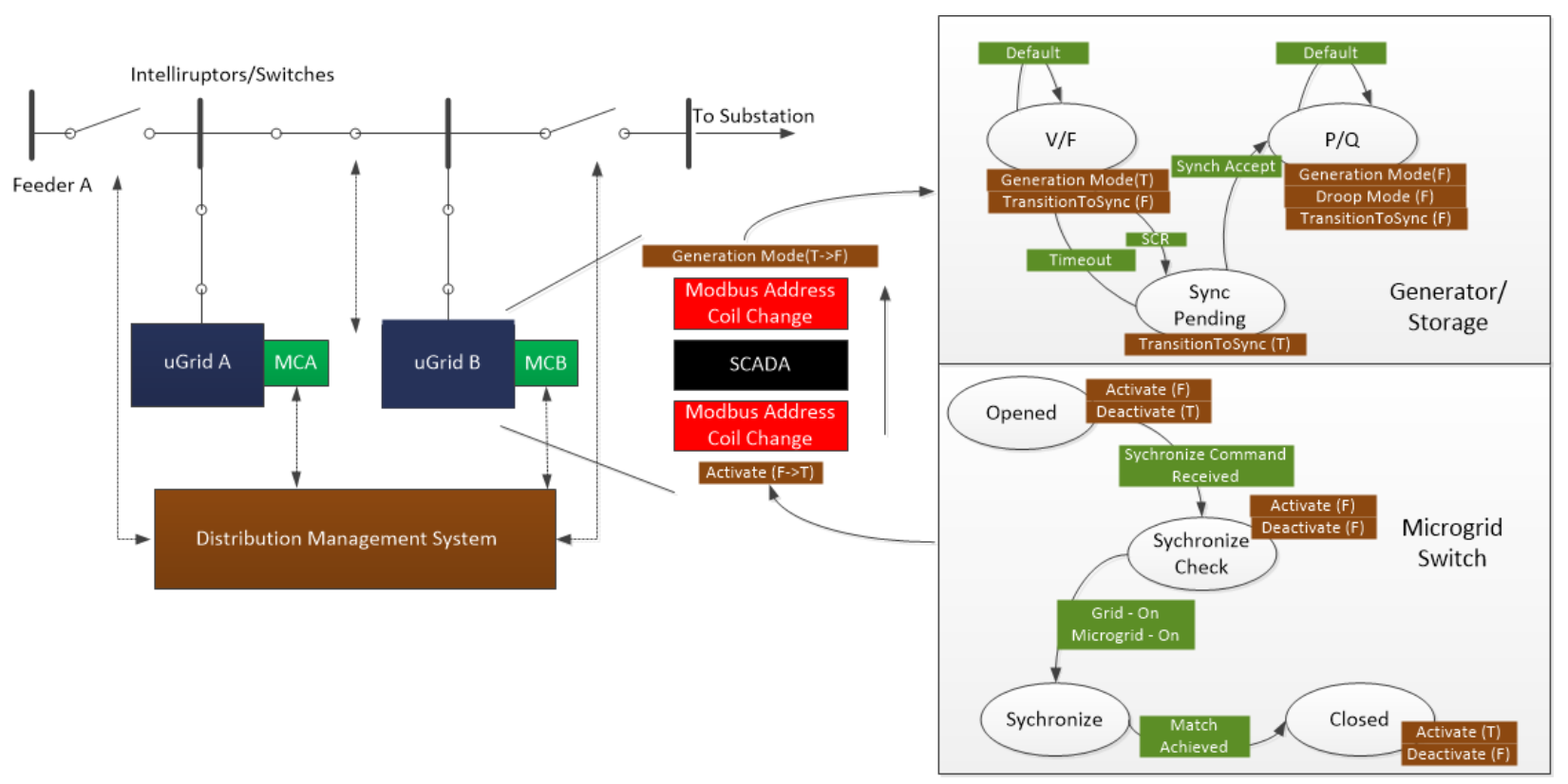

Figure 9. Example of resynchronization under hierarchy control architecture.

\subsubsection{Distributed Control Architecture}

Under hierarchical control architecture, DMS/ADMS only has direct control of feeder sectionalizing switches, while all microgrid PCCs are directly controlled by corresponding microgrid controllers. The microgrid controller determines the operation modes (grid-connected or islanded) and optimizes the resources within the microgrid. Nevertheless, DMS/ADMS can induce microgrids to provide certain services or support by distributing incentives to all subscribed microgrid controllers. Typical distributed control and communications architecture for networked microgrids control and management is shown in Figure 10. 
- In grid-connected mode, all microgrids are integrated directly with the distribution feeders or other grid-connected microgrids. DMS/ADMS collects measurements at PCC as well as the whole distribution grid, then generates and distributes well-designed incentive or price signals to microgrid controllers. For better economic performance, microgrid controllers would optimize their resources to provide corresponding services. DMS/ADMS would adjust the incentive signals based on the feedback from microgrids. This process would continue until Nash equilibrium is reached. From this point of view, an energy and ancillary services market on the distribution level needs to be designed. More extensive research is needed in this area.

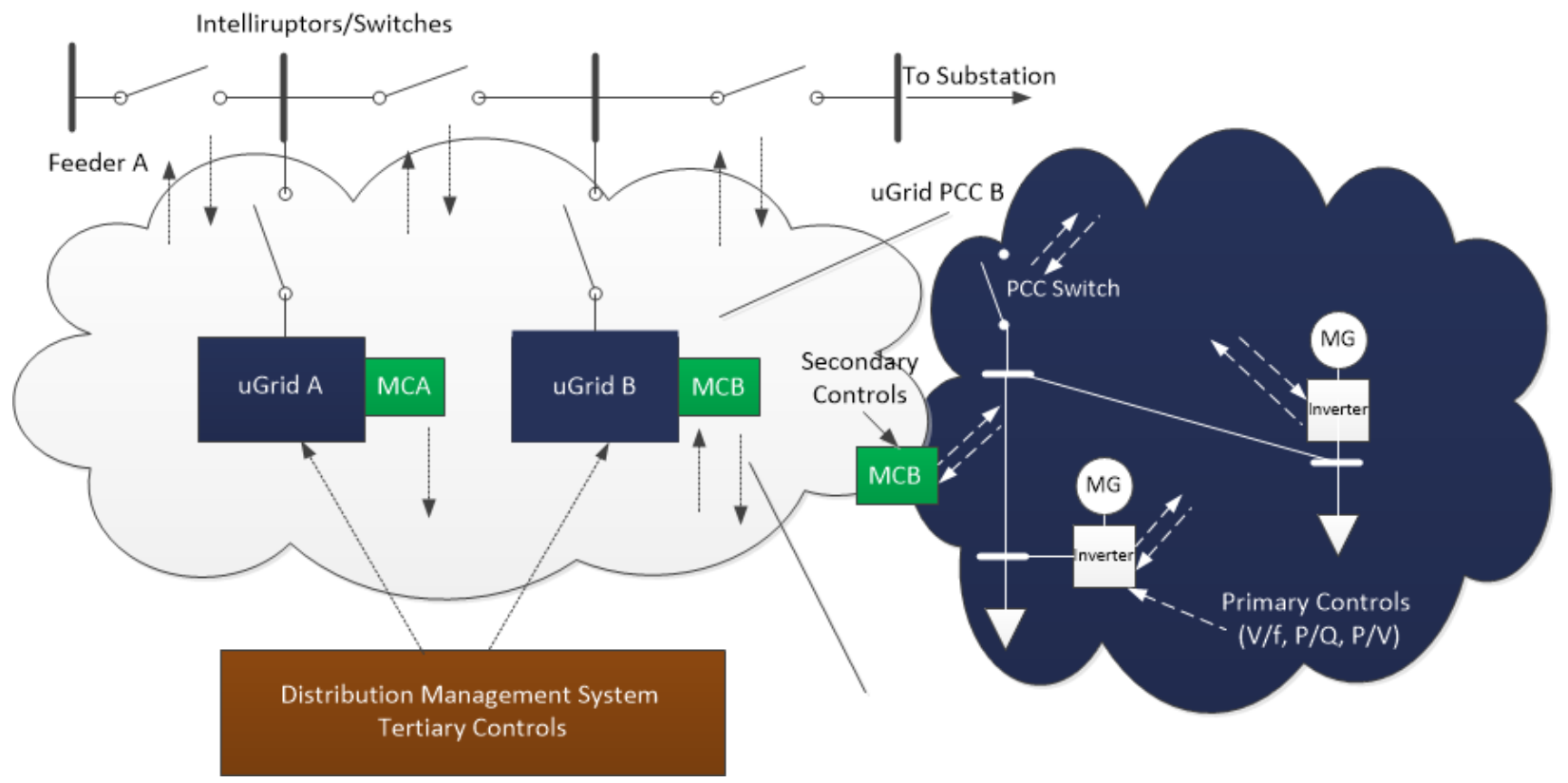

Figure 10. Distributed control with publish/subscribe communications.

- In islanding mode, each microgrid is required to have a capable V/f source for basic off-grid functionality with a separate microgrid controller. For both intentional and unintentional islanding, microgrid controllers don't need direct DMS/ADMS intervention, but microgrid controllers need to broadcast any intentional or unintentional islanding actions for safety issues. Other microgrid controllers receive this information and determine their need to island independently. DMS/ADMS may request that certain microgrids island with corresponding incentives.

- For the resynchronization process, microgrids should resynchronize to a grid-connected feeder as the first option for economic and reliability benefits. However, if a grid-connected feeder is not available at that moment, multiple neighboring microgrids may merge for better performance. The resynchronization process for these two options are:

- Resynchronization to grid-connected feeder as normal.

- Resynchronization to another islanded microgrid is more complicated. First, the interconnecting microgrid must publish a request to close interconnecting intertie. Then, two microgrids willing to be interconnected need to negotiate details such as who should provide the V/f source, what the power at voltage at PCC should be, etc. Once they reach an agreement, the interconnection PCC is closed and issues information directly to the V/f source for a state change (Figure 11). If there is no direct PCC switch connection between these microgrids, they can request that DMS/ADMS identify possible routes through available feeders. After both microgrids and DMS make an 
agreement for the interconnection, the resynchronization switch is closed, and two islanded microgrids are merged. The challenges of this kind of resynchronization include

- The microgrids that will be merged must have enough reserve and inertia to accommodate the new interconnected microgrid. The resynchronization onto a very weak grid (another microgrid) may impact the local voltage at the PCC enough to reinitiate an unintentional island based on settings.

- The microgrid controller should dynamically inherit resources (loads, DREs, switches, etc.) from feeders on the interconnection route or other microgrids. Because the interconnection of islanded microgrids may involve sections of feeders, the boundaries of microgrids are subject to change. This requires that the microgrid controller have plug-and-play capability.

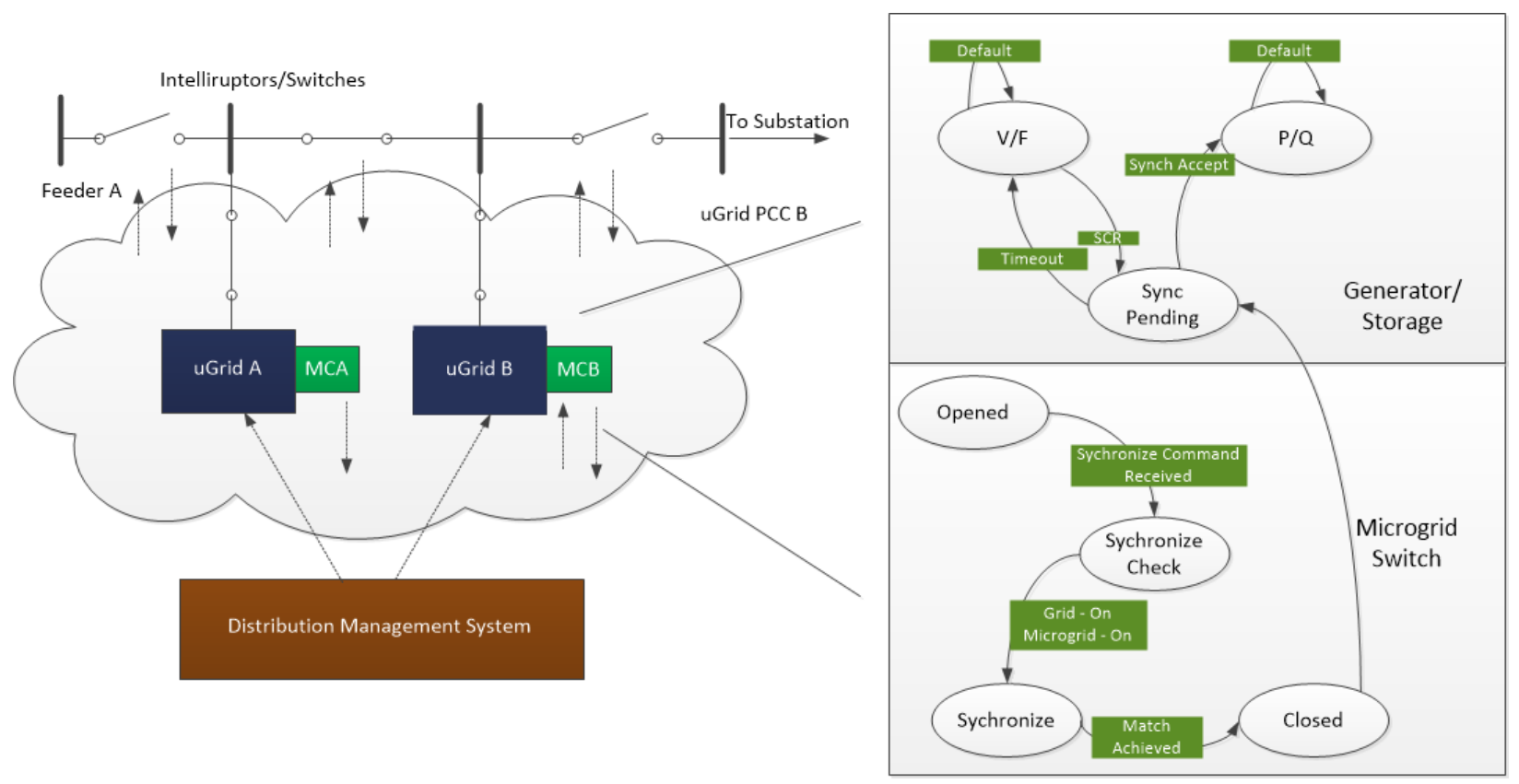

Figure 11. Example of resynchronization under distributed control architecture.

\subsection{BENEFITS}

\subsubsection{Benefits to Distribution System}

While the use of a microgrid as a source of resilience is a well-known benefit, there are other values from microgrid integration that could be realized. A microgrid optimizes with assets within the microgrid and can target any number of objective functions. With careful integration, microgrids integrated with utility distribution systems could be excellent resources for the distribution grid, interacting with DSOs as well as with other microgrids, as part of an integrated power delivery system from customer, to distribution utility, to transmission operator. These values are the key to widespread microgrid deployment.

The benefits to the distribution system fall into four categories:

- better uncertainty management,

- increased economic benefits and efficiency,

- improved power quality, and

- enhanced reliability and resiliency. 
First, microgrids could be viewed as an aggregator of DERs that can cope with intermittency challenges. With a DMS or third-party microgrid coordinator acting as the aggregator or master controller of distributed microgrids, the uncertainties of renewable energy resources, such as wind and PV from multiple microgrids, could be reduced by aggregating and correlating the information. Furthermore, microgrids that include energy storage systems could play an important role in smoothing intermittent renewable resources and load consumption impacts within the microgrid boundary.

With integration of multiple microgrids, the economy and efficiency of a distribution system could be significantly improved. At the distribution level, integrated distributed microgrids could reduce peak load, facilitate demand response, provide voltage/VAR support, reduce system loss, and even participate in CVR. At the transmission level, these same integrated microgrids would be able to increase the efficiency of the transmission resources and defer the investment of new transmission and substation infrastructure. Microgrid functionality could evolve to provide Independent System Operators (ISOs) with new integration options to distribution systems [8].

The power quality of a distribution system can be improved with integrated microgrids. Existing work has proven that with microgrid controls and optimization, overall voltage profiles of a distribution system can be improved with reduced over-voltage and under-voltage conditions [9]. Microgrids have also been shown to correct the power factor [10] and provide voltage and load balancing features across the three phases [11].

Resiliency is considered to be "the ability to prepare for and adapt to changing conditions and withstand and recover rapidly from disruptions [12]." Resiliency with respect to extreme events, such as a major hurricane, is considered one of the key features of smart distribution systems by the U.S. Department of Energy. One of the most effective measures to enhance distribution system resiliency is by using microgrids to restore as many loads as possible [13]. From the reliability point of view, both the System Average Interruption Frequency Index (SAIFI) and System Average Interruption Duration Index (SAIDI) can be reduced with microgrids [14].

\subsubsection{Benefits to Microgrids}

While the distribution system has been shown to benefit from support of multiple microgrids, a microgrid does benefit from the support of another microgrid. A microgrid connects to the distribution system or other microgrids at the PCC. The microgrid controller is the most critical component that rapidly controls power exchanges, generation, load, and energy storage and demand response to balance supply and demand within the microgrid and deliver the desired power exchange and functionality at the PCC. To optimize the operation of a microgrid, the microgrid controller must communicate and interact with other microgrids, DSOs and markets, in addition to communicating with the individual resources within the microgrid.

The benefits that microgrids receive from integrating with other microgrids or distribution systems fall into four categories:

- receiving V/f support from the distribution system,

- reducing operational cost and improving operational efficiency,

- facilitating high reliability for critical load, and

- maximizing benefit by participating in energy and ancillary service markets.

With integration to a distribution system or other microgrids, a microgrid can rely on the distribution system or a larger microgrid for frequency and voltage regulation. In addition, the uncertainties of renewable resources and volatile load will be covered by the distribution system. Thus, the tasks of 
voltage regulation, frequency regulation, and reserve management can be taken by the distribution system or another larger microgrid.

Operational efficiency can also be improved from two aspects. On one side, the capacity of reserve can be released to produce energy or reactive power as needed. On the other side, the PCC acts as an additional primary source or responsive load, which gives extra flexibility for the microgrid controller to schedule its own generation and load. In particular, microgrids with energy storage systems could arbitrage when the price at PCC is fluctuating.

During normal operation, the microgrids are connected with the distribution system, which manages the power flow, voltage, and frequency at PCC. Once the distribution system is down due to a disturbance, a microgrid has the ability to automatically island from the distribution system and continue serving the loads, especially critical loads within the microgrid. This significantly increases the service reliability for critical loads [15].

Finally, integrating with a distribution system or other microgrids provides a microgrid with a series of opportunities to participate in energy and ancillary service markets. A microgrid can sign a power purchase agreement (PPA) with other microgrids as well as with a distribution system. Provided a distribution energy and ancillary service market, a microgrid can submit bids of energy, reserve, frequency regulation, and reactive power, as well as other ancillary services.

\section{FUNCTIONAL REQUIREMENTS OF DMS AND MICROGRID CONTROLLER}

\subsection{REQUIREMENTS OF DMS}

With increasing penetration of DERs and microgrids into the distribution level, the traditional passive distribution network without any resources is gradually being transformed into an active system with both dispatchable and nondispatchable power sources [16]. Therefore, the usual "install and forget" principles have become less feasible and potentially could compromise operating efficiency. Within this context, new control and operation strategies capable of coordinating microgrids in various connection architectures efficiently to achieve operational objectives are in particular need of development [17], [18]. Some new modules, such as uncertainty aggregation, contingency analysis, fault management, etc., need to be developed and included in current DMS features. More commonly, current DMS modules, such as market bidding, volt/VAR control, restoration, reconfiguration, etc., need to be updated to consider the new characteristics of networked microgrids.

Table 1 is a list of detailed functional requirements for DMS integration of microgrids and DERs. These functions are sorted into five categories: energy management, protection and control, resiliency, ancillary services, and GUI and data management. Each category has a particular focus or objective:

1. The functions in energy management have the primary target of power balancing in steady state.

2. The functions in protection and control support V/f control.

3. The functions in resiliency aim to increase survivability of a microgrid under disturbances or severe weather conditions.

4. The functions in ancillary services strive to support interaction with the local utility or distribution system operator

5. The functions in data management address interoperability and data management. 
Table 1. Functional requirements for DMS integration of microgrids and DERs

\begin{tabular}{|c|c|c|c|c|}
\hline Energy management & $\begin{array}{c}\text { Protection and } \\
\text { control }\end{array}$ & Resiliency & $\begin{array}{c}\text { Ancillary } \\
\text { services }\end{array}$ & $\begin{array}{c}\text { GUI \& data } \\
\text { management }\end{array}$ \\
\hline $\begin{array}{l}\text { Power balancing } \\
\text { (UC/ED/OPF) }\end{array}$ & $\begin{array}{l}\text { Relay protection } \\
\text { coordination }\end{array}$ & $\begin{array}{l}\text { Coordinated } \\
\text { microgrids with } \\
\text { feeder reconfiguration }\end{array}$ & $\begin{array}{l}\text { Utility event } \\
\text { response }\end{array}$ & Visualization \\
\hline $\begin{array}{l}\text { Energy market } \\
\text { participation } \\
\text { (aggregated bidding) }\end{array}$ & $\begin{array}{l}\text { Volt/VAR/on-load tap } \\
\text { changers control } \\
\text { (intermittent DERs) }\end{array}$ & $\begin{array}{l}\text { Coordinated } \\
\text { microgrids with } \\
\text { system restoration }\end{array}$ & $\begin{array}{l}\text { Transmission \& } \\
\text { distribution } \\
\text { congestion } \\
\text { management }\end{array}$ & $\begin{array}{l}\text { Situational } \\
\text { awareness/alarms }\end{array}$ \\
\hline $\begin{array}{l}\text { State estimation / } \\
\text { network connectivity } \\
\text { analysis }\end{array}$ & $\begin{array}{l}\text { Fault management of } \\
\text { microgrids }\end{array}$ & & spinning reserve & \\
\hline $\begin{array}{l}\text { Uncertainty aggregation } \\
\text { and management }\end{array}$ & & & $\begin{array}{l}\text { Frequency/ } \\
\text { voltage } \\
\text { regulation }\end{array}$ & \\
\hline $\begin{array}{l}\text { Contingency analysis } \\
\text { (microgrid islanded) }\end{array}$ & & & $\begin{array}{l}\text { black start } \\
\text { capacity }\end{array}$ & \\
\hline
\end{tabular}

In the first group, an uncertainty aggregation model needs to be developed considering the correlation between renewable resources in multiple integrated microgrids. Then a bidding strategy based on the aggregated uncertainty should be developed to formulate the optimal bidding curves for the networked microgrids. The market clearing results will be used to revise the dispatch results of individual microgrids. Due to the strong interaction between active power and reactive power control in the distribution system, the energy trading and ancillary services need to be co-optimized simultaneously. To ensure that the reliability of the distribution system is not compromised upon islanding of multiplemicrogrids, a contingency analysis needs to be conducted as well. Due to various states and multiple PCCs, the network topology may be subject to frequent changes, so the state estimation of distribution system needs to consider the real-time network connectivity.

In the second group, the settings of protection devices in the distribution system need to consider the fault current contribution of microgrids and coordinate with microgrid switch settings. The integration of a microgrid that is able to inject or sink power from the distribution system impacts the trip settings of protection elements on a distribution feeder. For this reason, research into adaptive protection techniques has been of high interest. Yet another critical challenge arises when networked microgrids attempt to ride through faults. Uncontrolled power injection and fault current contributions may hazardously impact the main grid (e.g., violating the fault current limit of the grid) causing over-voltages, instability, and power quality problems. Meanwhile, disturbances in the utility grid would propagate to networked microgrids through synchronized electrical links and adversely impact microgrid end users. Also, integrated microgrids can contribute to the volt/VAR control functions of the DMS by generating or consuming reactive power at the PCC. Today's inverter-based microgrids have highly sensitive trip-off or deactivation settings for grid contingencies and are limited on fault current contribution. Should a high penetration of sensitive inverter-based assets exist in networked microgrids, a minor fault could lead to a sudden loss of a large number of DERs and microgrids. This would endanger power system security and increase the risk of a brownout or blackout. For this reason, the newly amended IEEE 1547 will allow a wider ride-through tolerance so that microgrids can continue injecting power despite excursions in grid voltages and frequency. To mitigate these impacts overall, a fault management system/function for integrated microgrids is needed. 
In the third group, the feeder reconfiguration function of the traditional DMS should consider integrated microgrid capabilities. With microgrids providing both the active and reactive power support, the feeder reconfiguration actions of the DMS may be avoided or delayed. Furthermore, with interlinkage of microgrid control options to the DMS, the feeder reconfiguration and restoration options should achieve increased performance with less power loss and increased load restoration.

In order to explore the flexibility of microgrid resources and responsive demands, various energy and ancillary service markets or similar regimes need to be designed. Services that microgrids can provide to distribution system operators or ISOs include PPAs, emergency event response, spinning reserve, frequency regulation, voltage regulation, congestion relief for distribution and/or transmission system, and black start capability. Corresponding markets need to be designed in order to explore these capabilities of microgrids. The individual microgrid controller needs to communicate with DMS, bid in markets, follow the market clearing results from DMS, and provide various services for the operation of the distribution system.

Finally, the visualization and alarming systems of the DMS will require a more dynamic build-out. The potential for reconfiguration, islanding, and resynchronization of microgrids will create a challenge with situational awareness. Disappearing loads and PCC state changes will likely trigger alarms and confusion without direct coordination with the DMS.

\subsection{REQUIREMENTS OF MICROGRID CONTROLLER}

Beside the basic islanding and resynchronization functions, a microgrid controller needs to be improved in many respects in order to be integrated with a distribution system and/or other microgrids. Table 2 lists the functional requirements for integration of a microgrid controller with a distribution system and/or other microgrids.

Table 2. Functional requirements for microgrid controller

\begin{tabular}{lllll}
\hline \multicolumn{1}{c}{$\begin{array}{c}\text { Energy } \\
\text { management }\end{array}$} & Protection and control & Resiliency & Ancillary service & $\begin{array}{c}\text { GUI \& data } \\
\text { management }\end{array}$ \\
\hline $\begin{array}{l}\text { Energy market } \\
\text { participation } \\
\text { (bidding) }\end{array}$ & Low voltage ride-through & Black start capacity & $\begin{array}{l}\text { Day-ahead/real- } \\
\text { time/emergency } \\
\text { demand response }\end{array}$ & $\begin{array}{l}\text { Interface with DMS } \\
\text { for price } \\
\text { information and } \\
\text { orders }\end{array}$ \\
& & $\begin{array}{l}\text { Splug-and-play } \\
\text { Low frequency ride- } \\
\text { through } \\
\text { Fault current control }\end{array}$ & Frequency/voltage & regulation \\
& & Phase balancing and \\
& & power factor support \\
\hline
\end{tabular}

Similar to the requirements of DMS, the microgrid controller functions are sorted into five categories: energy management, protection and control, resiliency, ancillary services, and GUI and data management.

In the first and third groups, various bidding functions for power and various ancillary services need to be developed. These ancillary services include demand response, spinning reserve, frequency regulation, voltage regulation, power factor correction, and phase balancing. While the microgrid can provide these features, the lack of a compensational approach is slowing the deployment of these systems. In the second group, low V/f ride-through capabilities of microgrids need to be developed 
so that microgrids can continue injecting power despite excursions in grid voltages and frequency. This is a critically important feature for the security and stability of a distribution system. Finally, a microgrid controller needs a high-speed interface with the DMS to obtain price and other reporting actions to the DMS. To inherit resources from a distribution grid or other microgrids, a microgrid controller should have plug-and-play capability.

\section{CONCLUSION}

This is an initial scoping study for the interconnection of multiple microgrids with an electrical distribution system. Various aspects of networked microgrids have been analyzed. First, three basic interconnection architectures of networked microgrids, including serial microgrids on a single feeder, parallel microgrids on a single feeder, and interconnected microgrids on multiple feeders, are proposed. These are followed by a presentation of the direct master-slave communication and publish and subscribe communication to describe how communications impact the control issues. Two basic control architectures, hierarchical and distributed control, are discussed and compared. Thus, the potential benefits to both distribution systems and microgrids from networked microgrids are discussed. To realize these benefits, the functional requirements of DMSs and microgrid controllers are presented.

When networked microgrids and distribution systems are coordinated, both the distribution systems and the networked microgrids benefit. A number of technical and regulatory issues need to be studied. Several microgrid research needs were determined in this scoping:

- Coordination of

- energy trading and ancillary service management

- microgrids/DERs with feeder reconfiguration/restoration

- protection scheme (from islanded individually to various subnetwork connections)

- Higher order power balancing of local imbalances to compensate for variable supplies of renewable energy

- Multi-objective operational optimization (peak shaving, loss minimization, voltage regulation) across several microgrids

- Aggregated uncertainty management

- Jurisdiction and dynamic boundaries among microgrid EMSs, networked microgrid EMSs, and DMSs

- Strong interaction between active power and reactive power schedules and controls

- Quantification and allocation of the benefits of networked microgrids

- Distribution energy market managed by DSO

- Distribution ancillary service market managed by DSO

- Pricing mechanism in distribution network and impact on microgrid economics and optimization

- Demand response incentive signal design 
- Defining communication topics for microgrid-to-microgrid communications

- Demonstrable networked microgrid communications

\section{REFERENCES}

[1] DOE (US Department of Energy). 2011. DOE Microgrid Workshop Report, Office of Electricity Delivery and Energy Reliability Smart Grid R\&D Program, August. Available online at http://energy.gov/sites/prod/files/Microgrid\%20Workshop\%20Report\%20August\%202011.pdf

[2] A. G. Madureira and J. A. Pecas Lopes, "Coordinated voltage support in distribution networks with distributed generation and microgrids," IET Renew. Power Gen., vol. 3, no. 4, pp. 439-454, Dec. 2009.

[3] S. Beer, T. Gomez, D. Dallinger, I. Momber, C. Marnay, M. Stadler, and J. Lai, "An economic analysis of used electric vehicle batteries integrated into commercial building microgrids," IEEE Trans. Smart Grid, vol. 3, no. 1, pp. 517-525, Mar. 2012.

[4] A. G. Tsikalakis and N. D. Hatziargyriou, "Centralized control for optimizing microgrids operation," IEEE Trans. Energy Convers., vol. 23, no. 1, pp. 241-248, Mar. 2008.

[5] C. Marnay, "Microgrids and heterogeneous power quality and reliability," in Proc. Power Convers. Conf. (PCC), pp. 629-634, 2007.

[6] M. Agrawal and A. Mittal, "Micro grid technological activities across the globe: A review," Int. J. Res. Rev. Appl. Sci., vol. 7,no. 2, pp. 147-152, May 2011.

[7] J. Hu, T. Zhang, S. Du and Y. Zhao, "An Overview on Analysis and Control of Micro-grid System," Int. J. of Cont. and Auto., vol. 8, no. 6, pp. 65-76, May 2015. [Online]. Available: http://dx.doi.org/10.14257/ijca.2015.8.6.08

[8] G. Liu, Y. Xu and K. Tomsovic, "Bidding Strategy for Microgrid in Day-ahead Market based on Hybrid Stochastic/Robust Optimization," IEEE Transactions on Smart Grid, Vol. 7, No. 1, pp. 227237, Jan. 2016.

[9] G. Liu, O. Ceylan, Y. Xu and K. Tomsovic "Optimal Voltage Regulation for Unbalanced Distribution Networks Considering Distributed Energy," Proceedings of the 2015 IEEE PES General Meeting, Denver, CO, Jul. 26-30, 2015.

[10] G. Liu, M. Starke, X. Zhang and K. Tomsovic “A MILP-Based Distribution Optimal Power Flow Model for Microgrid Operation," Proceedings of the 2016 IEEE PES General Meeting, Boston, MA, Jul. 17-21, 2016.

[11] G. Liu, O. Ceylan, M. Starke and K. Tomsovic, "Advanced Energy Storage Management in Distribution Network," Proceedings of the 49th Hawaii International Conference on System Sciences (HICSS-49), Kauai, HI, Jan. 5-8, 2016.

[12] Office of the Press Secretary of the White House, "Presidential Policy Directive 21 - Critical Infrastructure Security and Resilience," [Online]. Available: http://www.whitehouse.gov/the-pressoffice/2013/02/12/presidential-policy-directive-critical-infrastructure-security-and-resil 
[13] Y. Xu, C. C. Liu, K. P. Schneider and D. T. Ton, "Toward a resilient distribution system," Power \& Energy Society General Meeting, 2015 IEEE, Denver, CO, pp. 1-5, 2015.

[14] C. C. Liu, "Microgrids in Distribution System Restoration" Panel Session at IEEE PES GM 2015. [Online]. Available: http://www.ieee-pes.org/presentations/gm2015/PESGM2015P-001078.pdf

[15] M. Wang, and H. B. Gooi "Spinning reserve estimation in microgrids," IEEE Transactions on Power System, Vol. 26, No. 3, pp. 1164-1174, Aug. 2011 .

[16] J. A. P. Lopes, N. Hatziargyriou, J. Mutale, P. Djapic, and N. Jenkins, "Integrating distributed generation into electric power systems: A review of drivers, challenges and opportunities," Elect. Power Syst. Res., vol. 77, no. 9, pp. 1189-1203, 2007.

[17] J. A. P. Lopes, N. Hatziargyriou, J. Mutale, P. Djapic, and N. Jenkins, "Integrating distributed generation into electric power systems: A review of drivers, challenges and opportunities," Elect. Power Syst. Res., vol. 77, no. 9, pp. 1189-1203, 2007.

[18] Y. Zhu and K. Tomsovic, "Optimal Distribution Power Flow for Systems with Distributed Energy Resources,” Int. J. Elect. Power Energy Syst., vol. 29, no. 3, pp. 260-267, Mar. 2007. 
